\title{
BILINEAR WEIGHTED HARDY-TYPE INEQUALITIES IN DISCRETE AND $q$-CALCULUS FRAMEWORKS
}

\section{Pankaj Jain, Saikat Kanjilal, Guldarya E. Shambilova And VLADIMIR D. STEPANOV}

Abstract. We characterize Hardy inequality in weighted Lebesgue sequence spaces involving discrete bilinear Hardy operator $\left(\sum_{i=-\infty}^{n} a_{i}\right)\left(\sum_{i=-\infty}^{n} b_{i}\right)$ and then we apply this information to characterize the inequality with $q$-bilinear Hardy operator

$$
\mathscr{H}_{q}(f, g)(x):=\left(\int_{0}^{\infty} \chi_{(0, x]}(t) f(t) d_{q} t\right)\left(\int_{0}^{\infty} \chi_{(0, x]}(t) g(t) d_{q} t\right)
$$

for all possible indices of summation.

Mathematics subject classification (2010): 26D10, 46E35.

Keywords and phrases: Weighted Hardy inequality, bilinear Hardy-type inequalities, discrete Hardy inequality, $q$-calculus, Jackson integral.

\section{REFERENCES}

[1] A.O. Baiarystanov, L.E. Persson, S. Shaimardan and A. Temirkhanova, Some new Hardy-type inequalities in q-analysis, J. Math. Inequal. 10 (2016), 761-781.

[2] G. BenNetr, Some elementary inequalities, Quart. J. Math. Oxford Ser. (2). 38 (1987), 401-425.

[3] G. Bennet, Some elementary inequalities II, Quart. J. Math. Oxford Ser. (2). 39 (1988), 385-400.

[4] G. Bennett, Some elementary inequalities III, Quart. J. Math. Oxford Ser. (2). 42 (1991), 149-174.

[5] M. S. Braverman And V. D. Stepanov, On the discrete Hardy inequality, Bull. London Math. Soc. 26 (1994), 283-287.

[6] M.I. Aguilar Cañestro, P. Ortega Salvador and C. Ramírez Torreblanca, Weighted bilinear Hardy inequalities, J. Math. Anal. Appl. 387 (2012) 320-334.

[7] P. Cheung And V. KaC, Quantum calculus, Edwards Brothers, Inc., Ann Arbor, MI, USA, 2000.

[8] T. ERNST, A comprehensive treatment of q-calculus, Birkhäuser/Springer Basel AG, Basel, 2012.

[9] T. ERnst, A new method of q-calculus, Doctoral thesis, Uppsala university, 2002.

[10] A. Gogatishvili, P. Jain And S. Kanjilal, On bilinear Hardy inequality and corresponding geometric mean inequality, Ricerche di Mat., https://doi .org/10.1007/s11587-020-00536-2.

[11] K. -G. GROSSE-ERDMANN, The blocking technique, weighted mean operators and Hardy's inequality, Lecture Notes in Mathematics, 1679, (1998), Springer-Verlag: Berlin.

[12] G. H. Hardy, J.E. Littlewood and G. Polya, Inequalities, Reprint of the 1952 edition. Cambridge Mathematical Library. Cambridge University Press, Cambridge, 1988. xii +324 pp.

[13] F. H. JACKSON, On q-definite integrals, Quart. J. Pure Appl. Math. 41 (1910), 193-203.

[14] S. Kanjilal, L.E. Persson AND G. Shambilova, Equivalent integral conditions related to bilinear Hardy-type inequalities, Math. Inequal. Appl., 22:4 (2019), 1535-1548.

[15] M. KrePelA, Iterating bilinear Hardy inequalities, Proc. Edinb. Math. Soc. 60:4 (2017), 955-971.

[16] A. Kufner, L. Maligranda And L. -E. Persson, The Hardy inequality. About its history and some related results, Vydavatelsky Servis, Plzen, 2007. $162 \mathrm{pp.}$

[17] A. KUfNER, L.-E. PERSSON AND N. SAMKO, Weighted Inequalities of Hardy Type, Second Edition, World Scientific New Jersey, 2017.

[18] L. Maligranda, R. Oinarov And L.-E. Persson, On Hardy q-inequalities, Czechoslovak Math. J., 64:3 (2014), 659-682. 
[19] B. OpIC And A. Kufner, Hardy-Type Inequalities, Pitman Research Notes in Mathematics Series, 211, Longman Scientific and Technical, Harlow, 1990.

[20] D. V. Prokhorov, V. D. Stepanov and E. P. Ushakova, Hardy-Steklov integral operators: Part I, Proc. Steklov Inst. Math. 300, Suppl. 2 (2018), S1-S112.

[21] S. Shaimardan, Hardy-type inequalities quantum calculus, Doctoral thesis, Luleå University of Technology, 2018.

[22] V. D. Stepanov and G. E. Shambilova, On iterated and bilinear integral Hardy-type operators, Math. Inequal. Appl., 22:4 (2019), 1505-1533.

[23] V. D. Stepanov and E. P. Ushakova, Bilinear Hardy-type inequalities in weighted Lebesgue spaces, Nonlinear Studies, 26:4 (2019), 939-953. 